\title{
Structure and promoter analysis of murine CAD and ICAD genes
}

\author{
Kohki Kawane ${ }^{1,2}$, Hidehiro Fukuyama ${ }^{1,2}$, Masashi Adachi ${ }^{1,2}$, \\ Hideki Sakahira $^{1,2}$, Neal G. Copeland ${ }^{3}$, Debra J. Gilbert ${ }^{3}$, \\ Nancy A. Jenkin ${ }^{3}$ and Shigekazu Nagata*,1,2 \\ ${ }^{1}$ Department of Genetics, Osaka University Medical School, Osaka, Japan \\ ${ }^{2}$ Core Research for Evolutional Science and Technology, Japan Science and \\ Technology Corporation, 2-2 Yamada-oka, Suita, Osaka 565-0871, Japan \\ 3 Mammalian Genetics Laboratory, ABL-Basic Research Program, National \\ Cancer Institute-Frederick Cancer Research and Development Center, \\ Frederick, Maryland 21702, USA \\ * corresponding author: Shigekazu Nagata, Ph.D., Department of Genetics, \\ Osaka University Medical School B-3, 2-2 Yamada-oka, Suita, Osaka 565- \\ 0871, Japan. \\ tel: 81-6-6879-3310; fax: 81-6-6879-3319; \\ e-mail: nagata@genetic.med.osaka-u.ac.jp
}

Received: 20.4.99; revised: 16.5.99; accepted: 2.6.99

Edited by Y. Kuchino

\begin{abstract}
Caspase-activated DNase (CAD) degrades chromosomal DNA during apoptosis, whereas ICAD (inhibitor of CAD) inhibits the CAD's DNase by binding to it. Here, we describe the assignment of murine $C A D$ and ICAD genes to the distal part of murine chromosome 4. Molecular cloning and structural analysis indicated that $C A D$ and ICAD genes are comprised of 7 and 6 exons, respectively. Two different ICAD mRNAs coding for two forms of ICAD proteins (ICAD-S and ICAD-L) were found to be produced by alternative splicing of intron 5 . The CAD and ICAD mRNAs were detected ubiquitously in various murine tissues. Analyses of the promoter activity with a series of deletion mutants of their $5^{\prime}$ flanking regions indicated that a $190-b p 5^{\prime}$ flanking region of the CAD gene was sufficient to promote the transcription. Whereas, a 120-bp flanking region of ICAD gene was required to promote its transcription. These regions do not show similarity between $C A D$ and ICAD genes, suggesting that expression of $C A D$ and ICAD genes is regulated by different mechanisms.
\end{abstract}

Keywords: apoptosis; CAD; ICAD; alternative splicing; expression; promoter

Abbreviations: CAD, caspase-activated DNase; ICAD, inhibitor of CAD; DFF, DNA fragmentation factor; RFLP, restriction fragment length polymorphism

\section{Introduction}

Apoptosis is a cell death process that removes useless, harmful, or senescent cells from the body. ${ }^{1}$ This process is characterized morphologically by cell shrinkage, chromatin condensation, and formation of apoptotic bodies, ${ }^{2}$ and biochemically by degradation of chromosomal DNA into nucleosomal units. ${ }^{3}$ Most of the apoptotic stimuli activate caspases, which are cysteine proteases that cleave various substrates such as poly(ADP) ribose polymerase, lamin, and fodrin to promote the apoptotic process. ${ }^{4-7} \mathrm{We}$ and others recently showed that a DNase (CAD, caspase-activated DNase; also called CPAN, caspase activated nuclease, as well as DFF-40, DNA fragmentation factor-40) is activated during apoptosis, and cleaves the chromosomal DNA. ${ }^{8-12}$ In the growing, non-apoptotic cells, CAD is complexed with its inhibitor (ICAD, inhibitor of CAD; also called DFF-45) and predominantly localized in the cytoplasm. When cells are induced to die by apoptotic stimuli such as Fas ligand or the kinase inhibitor staurosporine, caspases are activated. The activated caspases cleave ICAD at two positions, which releases CAD from ICAD. ${ }^{12}$

Murine and human CADs are comprised of 344 and 338 amino acids, respectively, with $76 \%$ identity. ${ }^{8-11}$ Murine ICAD exists in two forms - long and short (ICAD-L and ICAD-S) which consist of 331 and 265 amino acids, respectively. ${ }^{8}$ Murine ICAD-L is $76 \%$ identical to human DFF $-45,{ }^{13}$ suggesting that murine ICAD is the murine counterpart of human DFF-45. In addition, CAD and ICAD have a homologous domain of about 100 amino acids at the N-terminus. ${ }^{11} \mathrm{~A}$ similar domain has also been found in several proteins of unknown function. ${ }^{14}$

In this report, we showed that the murine $C A D$ and ICAD genes are localized close to one another on murine chromosome 4. The isolation and structural analysis of their chromosomal genes indicated that $C A D$ and ICAD comprise about 11 and $16 \mathrm{~kb}$ DNA, and are split by 6 and 5 introns, respectively. A comparison of the ICAD chromosomal gene with its mRNAs indicated that the ICAD- $L$ and ICAD-S mRNAs are generated by alternative splicing of intron 5. The $C A D$ and $I C A D$ genes are expressed ubiquitously in similar tissues. However, the $5^{\prime}$ flanking sequences of $C A D$ and $I C A D$ genes necessary to promote the transcription were not well conserved between the two genes, suggesting that the expression of the $C A D$ and ICAD genes is regulated differently.

\section{Results}

\section{Assignment of CAD and ICAD genes on murine chromosome}

The murine chromosomal location of Icad and Cad was determined by interspecific backcross analysis using progeny derived from matings of [(C57BL/6J x Mus spretus) $\left.\mathrm{F}_{1} \times \mathrm{C} 57 \mathrm{BL} / 6 \mathrm{~J}\right]$ mice. This interspecific backcross mapping panel has been typed for over 2700 loci that are well distributed among all the autosomes as well as the $X$ chromosome. ${ }^{15} \mathrm{C} 57 \mathrm{BL} / 6 \mathrm{~J}$ and $M$. spretus DNAs were 
digested with several enzymes and analyzed by Southern blot hybridization for informative restriction fragment length polymorphisms (RFLPs) using murine cDNA probes. The 5.3 and $3.8 \mathrm{~kb}$ Sphl M. spretus RFLPS (see Materials and Methods) were used to follow the segregation of the Icad locus and the 15.0 and $2.6 \mathrm{~kb} B g / l$ fragments were used to follow the Cad locus in backcross mice. The mapping results indicated that the two genes are linked and are located in the distal region of murine chromosome 4 linked to Htrld, Nppa and Gnb1. Although 100 mice were analyzed for every marker and are shown in the segregation analysis (Figure 1), up to 178 mice were typed for some pairs of markers. Each locus was analyzed in pairwise combinations for recombination
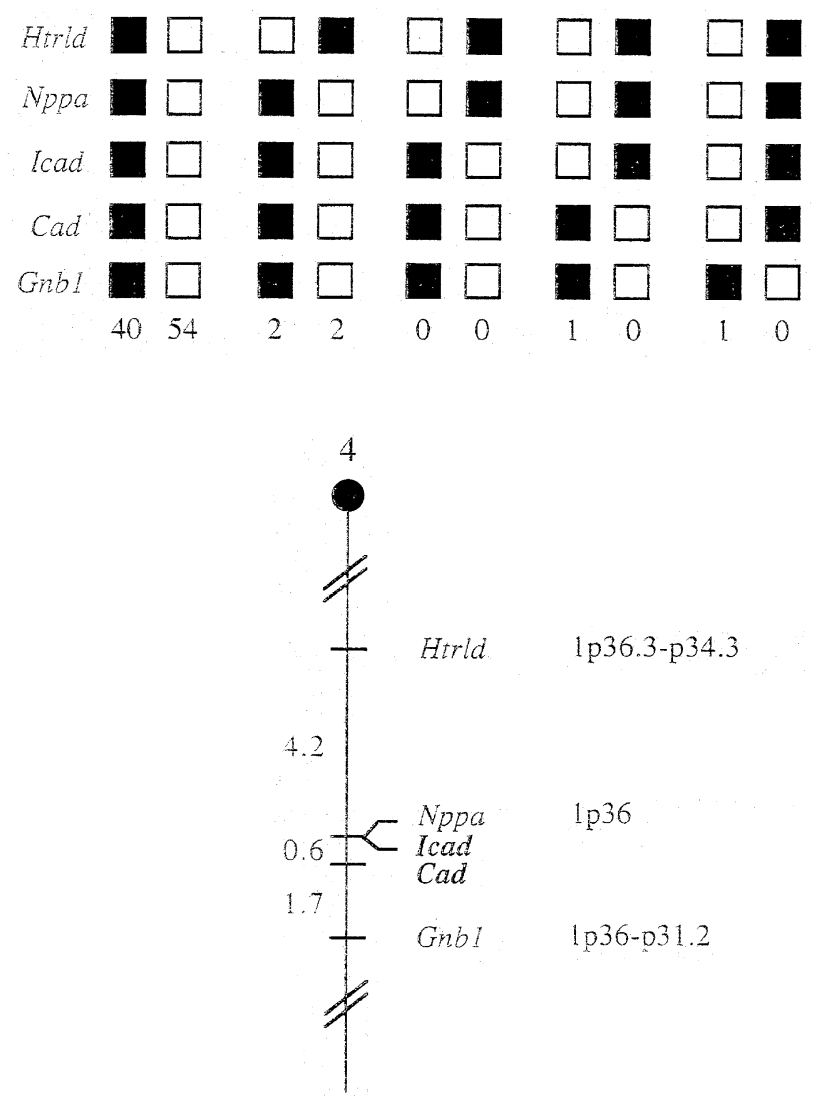

Figure $1 \mathrm{lcad}$ and Cad map in the distal region of murine chromosome 4. Icad and $\mathrm{Cad}$ were placed on murine chromosome 4 by interspecific backcross analysis. The segregation patterns of Icad and $\mathrm{Cad}$ and flanking genes in 100 backcross animals that were typed for all loci are shown at the top of the figure. For individual pairs of loci, more than 100 animals were typed (see text). Each column represents the chromosome identified in the backcross progeny that was inherited from the (C57BL/6J x M. spretus) $\mathrm{F}_{1}$ parent. The shaded boxes represent the presence of a $C 57 \mathrm{BL} / 6 \mathrm{~J}$ allele and white boxes represent the presence of a $M$. spretus allele. The number of offspring inheriting each type of chromosome is listed at the bottom of each column. A partial chromosome 4 linkage map showing the location of Icad and Cad in relation to linked genes is shown at the bottom of the figure. Recombination distances between loci in centimorgans are shown to the left of the chromosome and the positions of loci in human chromosomes, where known, are shown to the right. References for the human map positions of loci cited in this study can be obtained from GDB (Genome Data Base), a computerized database of human linkage information maintained by The William H. Welch Medical Library of The Johns Hopkins University (Baltimore, MD, USA) frequencies using the additional data. The ratios of the total number of mice exhibiting recombinant chromosomes to the total number of mice analyzed for each pair of loci and the most likely gene order are: centromere- Htrld-5/119-Nppa-0/ 109-Icad-1/160-Cad-3/178-Gnb1. The recombination frequencies [expressed as genetic distances in centiMorgans $(\mathrm{cM}) \pm$ the standard error] are $-H$ trld-4.2 $\pm 1.8-[N p p a, I c a d]-$ $0.6 \pm 0.6-C a d-1.7 \pm 1.0-G n b 1$. No recombinants were detected between Nppa and Icad in 109 animals typed in common suggesting that the two loci are within $2.8 \mathrm{cM}$ of each other (upper 95\% confidence limit).

\section{Chromosomal gene structure of the murine CAD and ICAD genes}

To determine the extron-intron organization of $C A D$ and $I C A D$, DNA fragments carrying the $C A D$ and ICAD chromosomal genes were isolated from a murine genomic library. Restriction-enzyme mapping and PCR analyses of these DNA fragments indicated the clones $\mathrm{Q} 9$ and $\mathrm{P} 1$ contain the entire $C A D$ and $I C A D$ chromosomal genes, respectively. A comparison of the genomic sequences with that of the $C A D$ CDNA indicated that the murine $C A D$ chromosomal gene consists of about $11 \mathrm{~kb}$, and is split by six introns (Figure 2A). The ICAD chromosomal gene was found to consist of about $16 \mathrm{~kb}$, and to be split by five introns (Figure 2B). The nucleotide sequences of the coding region of chromosomal $C A D$ was completely identical to the $C A D$ cDNA sequence. The nucleotide sequence of the ICAD chromosomal gene carried two silent mutations at positions $249(\mathrm{G}-\mathrm{A})$ and 378 $(T-C)$. All of the splice donor and acceptor sites conformed to the GT-AG rule for nucleotides immediately flanking exon borders. ${ }^{16}$ The $\mathrm{N}$-terminal region of about 100 amino acids is homologous between $\mathrm{CAD}$ and ICAD. ${ }^{11}$ This region was encoded by two exons in both $C A D$ and $I C A D$.

There are two isoforms for murine ICAD mRNA (ICAD- $L$ and ICAD-S), which code for proteins of 331 and 265 amino acids, respectively. ${ }^{8} I C A D-S$ is the same as ICAD-L truncated at the 261 amino acid position, and carries an extra 4 amino acids at the C-terminus. The $3^{\prime}$ non-coding region of the ICAD-S mRNA diverges from that of ICAD- $L$ mRNA. A comparison of the nucleotide sequence of the $I C A D$ chromosomal gene with ICAD-L and ICAD-S cDNAs indicated that ICAD-L mRNA contains exons 1-6. In contrast, the ICAD-S mRNA contains only exon 1-5, and its sequence continues into intron 5 (Figure 2C).

\section{Expression of CAD and ICAD mRNAs in murine tissues}

The tissue distribution of the $C A D$ and ICAD mRNAs was examined by Northern hybridization. As shown in Figure $3 A$, most tissues were found to express an mRNA of about $2.5 \mathrm{~kb}$ that hybridized with the $C A D$ probe. Some tissues such as spleen, thymus, small intestine and lymph nodes expressed a high level of $C A D$ mRNA. The skeletal muscle also showed a strong band hybridizing with $C A D$ probe, but its size (about $1 \mathrm{~kb}$ ) was significantly smaller than that found in other tissues. When Northern hybridization was carried out with the coding sequence of ICAD cDNA, two bands of 3.8 and $2.3 \mathrm{~kb}$ were 
detected in various tissues, particularly in the spleen, thymus and prostate (Figure 3B). The upper $3.8 \mathrm{~kb}$ mRNA hybridized with the $3^{\prime}$ non-coding regions of ICAD-S and ICAD-L CDNAs (data not shown). While, the lower $2.3 \mathrm{~kb}$ mRNA hybridized only with the $3^{\prime}$ non-coding region of $I C A D-L$ cDNA. The length of intron 5 is about $1.5 \mathrm{~kb}$, which agrees with the difference in length between the upper and lower ICAD
mRNAs. These results suggest that the upper band of $3.8 \mathrm{~kb}$ is the mRNA for ICAD-S, and carries the unspliced intron 5 . Whereas, the lower band of $2.3 \mathrm{~kb}$ represents the ICAD-L mRNA in which intron 5 is spliced out. The similar intensity of the upper and lower bands hybridizing with the ICAD probe (Figure $3 \mathrm{~b}$ ) indicates that $I C A D-S$ and $I C A D-L$ mRNAs are expressed at a similar level in most tissues.

(A) $\mathrm{CAD}$

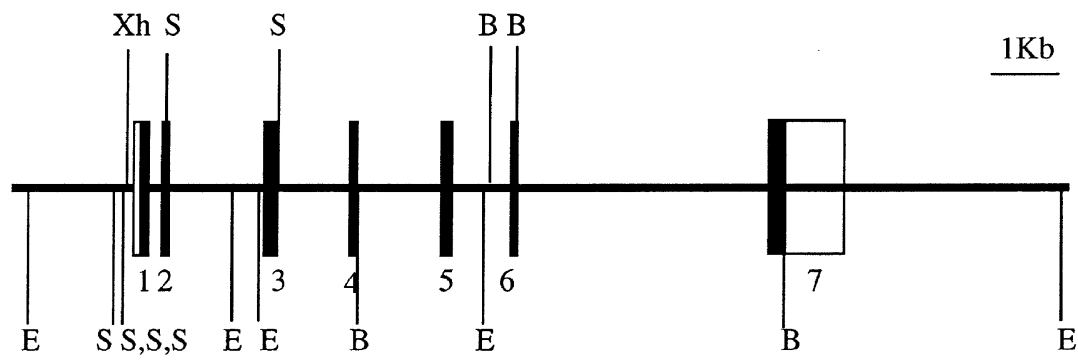

(B) ICAD

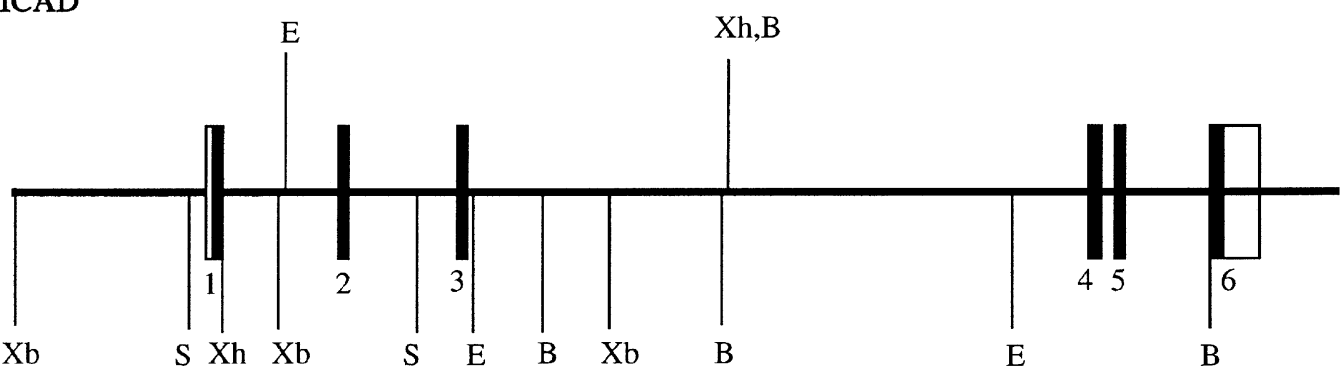

(C)

ICAD-L mRNA

$2.3 \mathrm{~kb}$

$123 \quad 4 \quad 56$

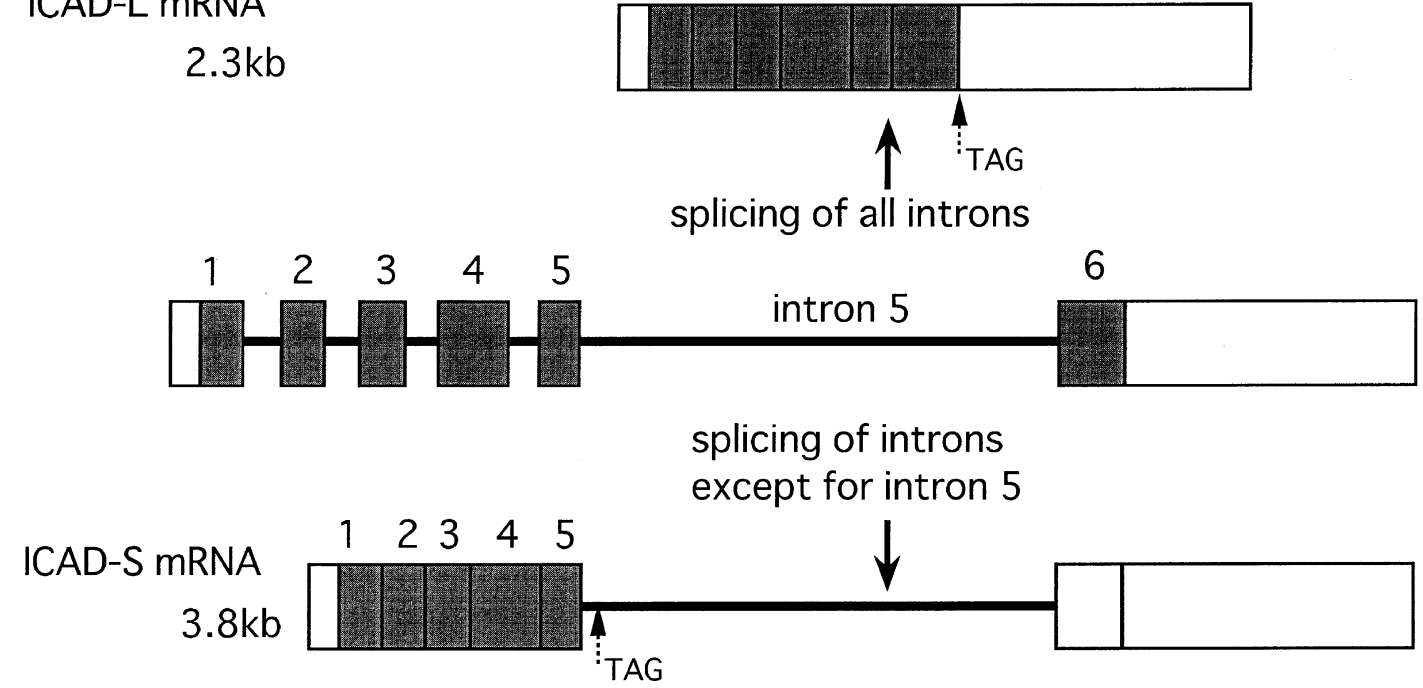

Figure 2 Chromosomal gene structures for the $C A D$ and ICAD genes. ( $\mathrm{A}$ and $\mathbf{B}$ ) The organization of the $C A D$ and ICAD chromosomal genes. The structures of $C A D(A)$ and ICAD (B) are schematically shown. The boxes and lines between them represent exons and introns, respectively. The filled areas represent the coding regions, while the non-coding regions are indicated by open areas. The restriction enzyme sites for BamHI (B), EcoRI (E), Smal (S), and Xhol (Xh) on the both genes, and for Xbal $(\mathrm{Xb})$ on the ICAD gene are shown. (C) Generation of two isoforms of ICAD mRNAs, ICAD-L and ICAD-S. A close-up view of the ICAD precursor RNA (middle), ICAD-L mRNA (top) and ICAD-S mRNA (bottom) are shown. Boxes represent exons, which are numbered above boxes. The shaded and open areas represent the coding and non-coding regions, respectively. The precursor RNA undergoes splicing of intron 5 to produce ICAD-L mRNA, while intron 5 remains unspliced to generate ICAD-S mRNA. The positions of the first termination codon are indicated by arrows 


\section{The promoter sequence of ICAD and CAD chromosomal genes}

The $5^{\prime}$ flanking sequences (660 and $730 \mathrm{bp}$, respectively) of the murine $C A D$ and $I C A D$ genes are shown in Figure $4 A$ and B. To determine the transcription initiation sites for $C A D$ and ICAD mRNAs, 5'-RACE procedure was carried out with mRNA from murine WR19L T cell lymphoma cells. As shown in Figure 4C, PCR with an anti-sense primer at 371 nucleotides downstream of the ATG initiation codon in the $C A D$ cDNA produced a major band of $460 \mathrm{bp}$. The same procedure for ICAD mRNA with an antisense primer at 614 nucleotides downstream of the ATG codon gave a major band of $740 \mathrm{bp}$. The major PCR products for $C A D$ and ICAD mRNAs were then subcloned and sequenced. Most of the PCR products for $C A D$ mRNA started around the $G$ at position $+1,43$ nucleotides upstream from the initiation codon, while minor PCR products (less than 10\%) started from the $G$ at position -37 (Figure 4A). On the other hand, most of the PCR products for ICAD mRNA started from the $A$ at position $+1,88$ nucleotides upstream from the initiation codon (Figure 4B). These bases were therefore tentatively assigned as the transcription initiation sites for $C A D$ and ICAD mRNAs. There is no 'TATA-like' box immediately upstream of the transcription initiation site in either the CAD or ICAD gene. A sequence of about 500 nucleotides up to position -600 in the $C A D$ gene is extremely rich in $\mathrm{G}$ and $\mathrm{C}$ nucleotides $(75 \% \mathrm{G} / \mathrm{C})$. In contrast, the $5^{\prime}$ promoter region of the ICAD gene was rich in $\mathrm{A}$ and T nucleotides $(42 \% \mathrm{G} / \mathrm{C})$. There was a B1-like repetitive sequence of about $340 \mathrm{bp}$ at 320 nucleotides upstream of the transcription initiation site of the ICAD gene.

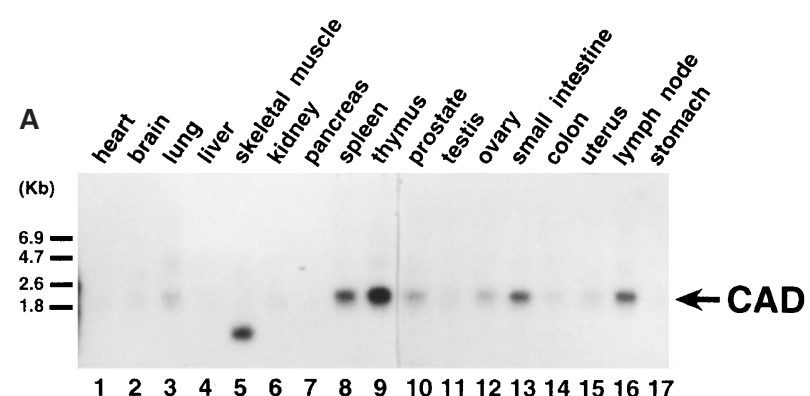

B

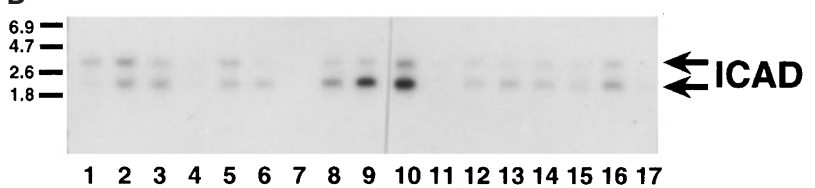

C

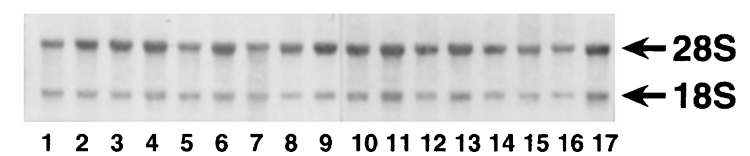

Figure 3 Expression of the $C A D$ and ICAD mRNAs in murine tissues. Poly(A) RNA was prepared from the indicated tissues by using the Quick Prep ${ }^{T M}$ micro mRNA preparation kit (Pharmacia). Two micrograms of RNA from each source was subjected to electrophoresis and analyzed by Northern hybridization as described previously. ${ }^{28}{ }^{32} \mathrm{P}$-labeled murine $C A D$ CDNA (A) or ICAD-L CDNA (B) was used as the probe. The filter was stained with methylene blue, and is shown in (C). The positions of $28 \mathrm{~S}$ and $18 \mathrm{~S}$ rRNAs are indicated by arrows on the right

\section{Promoter analysis of CAD and ICAD genes in human HeLa and Jurkat cells}

To examine the promoter activity of the $5^{\prime}$ flanking region of $C A D$ and ICAD genes, a 544-bp fragment carrying the sequence of -590 to -46 of $C A D$ gene or a $297-\mathrm{bp}$ fragment carrying the sequence of -305 to -8 of ICAD gene was introduced into the upstream of the luciferase reporter gene (pBPA). As shown in Figure 5A, compared with the basic promoter in $\mathrm{PBPA}$, the flanking region of $C A D$ gene in PCADP stimulated transcription of the luciferase gene about 120 or 25 times in HeLa and Jurkat cells, respectively. When a longer fragment $(4.7 \mathrm{~kb})$ carrying the $5^{\prime}$ upstream flanking region was used to drive the reporter gene, the luciferase activity in the transfected cells was comparable to that found with pCADP, suggesting the control element(s) responsible for the $C A D$ gene transcription is within the $554 \mathrm{bp}$ fragment in pCADP. To determine regulatory elements in this region, a series of deletion mutants in the $5^{\prime}$ flanking region of the $C A D$ gene was then joined to luciferase gene, and the promoter activity was assayed. As shown in Figure $5 \mathrm{~A}$, deletion of the sequence from -590 to -190 had no significant effect on the promoter activity. On the other hand, the deletion of the sequence from -189 to -133 caused a sixfold or threefold reduction of the promoter activity in HeLa and Jurkat cells, respectively. These results suggest that a transcriptional regulatory element(s) are on the region from -189 to -133 of the CAD gene.

The 297-bp DNA fragment of the $5^{\prime}$ flanking region of ICAD gene in pICADP plasmid also stimulated transcription of the luciferase gene about 100 times and 30 times in HeLa and Jurkat cells, respectively (Figure 5B). Activation of transcription by a longer fragment containing the sequence up to $3.7 \mathrm{~kb}$ was similar to that obtained with the 297-bp fragment (data not shown), suggesting that the transcription of ICAD gene is controlled by the 297-bp fragment in the upstream of the transcription initiation site. Deletion of the sequence from -305 to -119 had little effect on the promoter activity. Further deletion up to -80 caused a significant reduction of the promoter activity in HeLa cells, although its effect in Jurkat cells were less significant. Whereas, when the sequence was further deleted up to -56 , the promoter activity dropped in both HeLa and Jurkat cells.

\section{Discussion}

Murine and human CAD have an identity of $76 \%$ at the amino acid sequence level. We previously showed that there are intact and processed pseudo genes for human $C A D .^{11}$ The intact human $C A D$ gene is localized on the short arm of chromosome 1 (1p36.3).${ }^{11}$ Here, we cloned a single introncontaining gene for murine $C A D$, and assigned it to the distal part of murine chromosome 4 , which is syntenic to the short arm of human chromosome 1 (1p36). These results suggest that there is a single functional gene for $C A D$ per murine haploid genome. Murine ICAD inhibits the CAD's DNase activity, and has an identity of $76.1 \%$ with human DFF- 45 , one of the subunits of DNA fragmentation factor in apoptosis. ${ }^{13} \mathrm{We}$ 
cloned an intron-containing gene for murine $I C A D$, and showed that it is closely localized with the $C A D$ gene on murine chromosome 4. Human DFF-45 was recently assigned to the region of $1 \mathrm{p} 36.3,{ }^{17}$ where the $C A D$ gene is located. ${ }^{11}$ These
(A) CAD

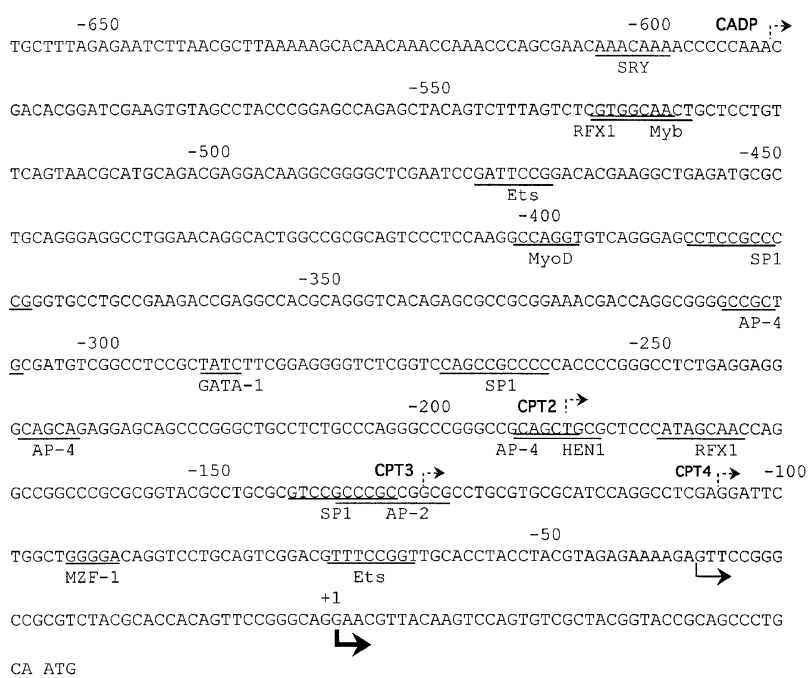

(B) ICAD

$$
-700
$$

GCTTGTAAATCATATCCTTTAAACTTCAAAAGTCACAAAACCAGTCCTGAAAAACACACAAACACACACA $-650$

$-600$ CACACAATTTTCTCTTTTATGTAGCTCTGGCTGTGTAGAAACTCACTATGTAGACCAGGCTGCCCTGGAA $-550$ CTCATAAAGATCCTTCTGCCTCCTGAGGATTAAAGGCCTGAGCCACAACACCCAGTTCAATGGCAACTCT TAAAACATGAATATGCATCTGGAGAGATGGCCCAGCAGTTAAGAGCACTGACTGCTCTTCCAAAGGTCCT $-400$ GAGTTCAAATCCCAGCAACCACATGATGGCTCACAACCATCCGTAATGAGATCTGATGCCTTCTTCTGGT $-350$ GTGTCTGAGGACAGCTACAGTGTACTTATGTATAAATAAATCTTTAAAAAGGAAAAAAAAAAAACATGAA ICADP : $\rightarrow-300 \quad-250$ TATATGGCTGGCAATGTAGTGTAGAACTATGCTTACCATACATGAGACCCCGGGTTTTCCATCATACAGT

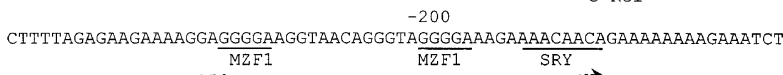

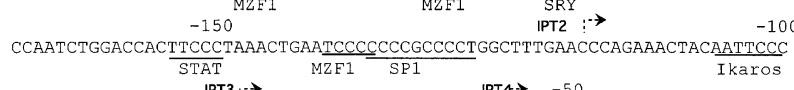
IPT3:- $\rightarrow$

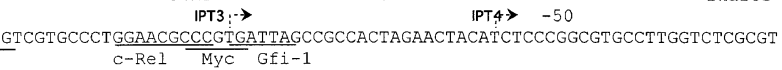
AACTTGCTGAGAGAACTACATCTCCCGTCAGGCAGCGGGAGGAAGGTTGTCAAAGCTTAGGGAAGCGGCT CTGGGCTACCTGGGATTTCCCAGAGTCGCCAGGTCCCCACTTTGAGG ATG

(C)
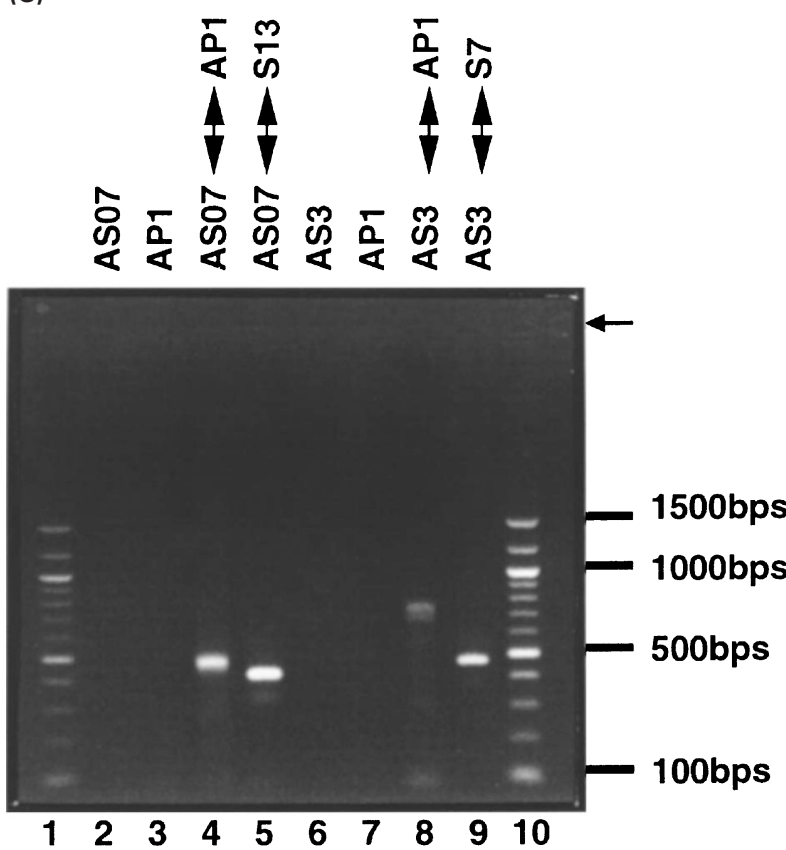

Figure 4 The $5^{\prime}$ flanking region of the $C A D$ and $I C A D$ chromosomal genes. ( $\mathbf{A}$ and $\mathbf{B}$ ) Nucleotide sequence of the $5^{\prime}$ flanking region of murine $C A D(\mathbf{A})$ and $I C A D$ (B) chromosomal genes. The nucleotide sequence is numbered from the major transcription initiation site. The major transcription initiation site is indicated by a bold arrow, while the minor initiation site is indicated by a thin arrow. The B1-like repetitive sequence in ICAD gene is doubly underlined. The binding sites for transcription factors were identified with MatInd and MatInspector programs, ${ }^{29}$ and are underlined. (C) $5^{\prime}$ RACE procedure for the CAD and ICAD mRNAs. Poly(A) RNA from murine WR19L cells were reverse-transcribed with an anti-sense primer specific for $C A D$ (lanes 2-5) or ICAD (lanes 6-9), and subjected to the $5^{\prime}$ RACE procedure as described in Materials and Methods. PCR was carried out with a specific anti-sense primer (AS07 for CAD and AS3 for ICAD) and a sense primer (AP1) in the adaptor (lanes 4 and 8). As controls, PCR was carried out with the anti-sense primer alone (lanes 2 and 6 ) or the sense adaptor primer alone (lanes 3 and 7). PCR was also carried out with the sense and anti-sense primers (AS07 and S13 for CAD, and AS3 and S7 for ICAD) that are specific to the CAD or ICAD mRNA, respectively (lanes 5 and 9). The DNA size marker (100 bp DNA ladder, New England Biolabs) was subjected to electrophoresis in parallel, and the size is shown on the right in bp. An arrow indicates the origin of electrophoresis 


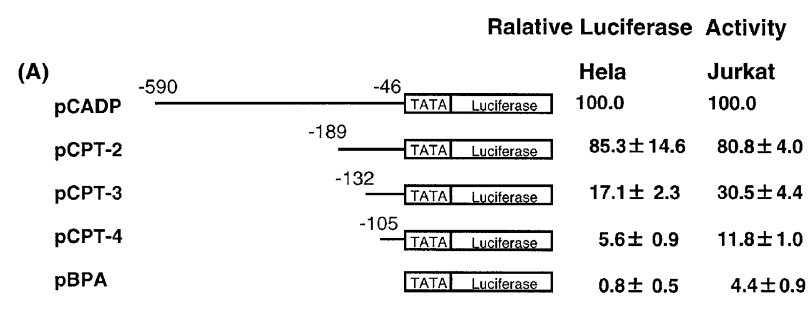

(B)

\begin{tabular}{|c|c|c|c|c|}
\hline & -305 & & Hela & Jurkat \\
\hline pICADP & & & 100.0 & 100.0 \\
\hline pIPT-2 & & \begin{tabular}{|l|l|} 
TATA Luciferase \\
\end{tabular} & $92.3 \pm 12.6$ & $80.5 \pm 9.9$ \\
\hline pIPT-3 & & -79 TATA Luciferase & $26.9 \pm 3.4$ & $67.0 \pm 8.1$ \\
\hline IPT- & & -55 - TATAL Lucifierase & $3.4 \pm 0.1$ & $24.7 \pm 2.0$ \\
\hline BPA & & \begin{tabular}{|l|l|} 
TATA & Luciferase \\
\end{tabular} & $0.2 \pm 0.2$ & $3.3 \pm 0.9$ \\
\hline
\end{tabular}

Figure 5 Promoter activities of $5^{\prime}$ flanking region of $C A D$ and ICAD genes. The promoter regions of $C A D(A)$ and ICAD (B) genes connected to the luciferase gene are shown by horizontal bars. The numbers above each bar indicate positions from the major transcription initiation site. TATA and Luciferase indicate the TATA box, and firefly luciferase gene, respectively. BPA represents pBPA-TATALuc vector. ${ }^{27}$ The promoter activity of each construct was determined in HeLa and Jurkat cells, and relative luciferase activities are expressed as a percentage of that of pCADP (A) or pICADP (B), respectively. Transfection was done four times, and the average values are shown with standard deviation

results suggest that human $D F F-45$ is the human ortholog of $I C A D$, and that there is a single chromosomal gene for ICAD per murine haploid genome. We have compared our interspecific map of chromosome 4 with a composite murine linkage map that reports the map location of many uncloned murine mutations (provided from Murine Genome Database, a computerized database maintained at The Jackson Laboratory, Bar Harbor, ME, USA). Icad and Cad were mapped in a region of the composite map that lacks murine mtuations with a phenotype that might be expected for an alteration in these loci (data now shown).

ICAD, which is more abundant than CAD, can bind to CAD. ${ }^{8,12}$ In growing cells, CAD exists as a complex with ICAD, ${ }^{10,13,18}$ suggesting that CAD and ICAD function as subunits. The N-terminal domain of about 100 amino acids is homologous between CAD and ICAD. However, no homology is apparent between the other parts of these molecules. ${ }^{11}$ Nonetheless, $C A D$ and $I C A D$ are localized in the same chromosomal region in human and mouse. Since functional CAD cannot be synthesized without ICAD,, 18 $C A D$ and ICAD may have evolved as a set of genes. To further examine this possibility, it would be necessary to determine the detailed arrangement of the $C A D$ and ICAD genes on human and murine chromosomes, as well as on the chromosomes of other organisms. The $\mathrm{N}$-terminal homologous domain of CAD and ICAD is coded by two exons in both genes, and introns in this domain are inserted at similar positions in the $C A D$ and ICAD genes. However, intron 1 is inserted after the third letter of a codon in the $C A D$ gene, while it is inserted after the first letter of a codon in the ICAD gene, suggesting that this domain may not have derived from a common ancestral gene. Rather, the sequence encoding the $\mathrm{N}$-terminal portions of $C A D$ and $I C A D$ may have converged from different ancestral genes to a similar sequence to acquire a similar function. Although the function of the $\mathrm{N}$-terminal domain of CAD and ICAD has not yet been elucidated, it is possible that it works as a homophilic interacting domain as found for the death and CARD domains. ${ }^{19-21}$

Structural analysis of the murine ICAD gene indicated that two different mRNAs for ICAD (ICAD-L and ICAD-S) are generated by alternative splicing of intron 5. ICAD-L and ICAD-S mRNAs were expressed at a similar level in various murine tissues. Equal amounts of ICAD-S and ICAD-L proteins were also detected by Western blotting in human and murine cell lines, ${ }^{18,22}$ suggesting that the human ICAD gene also produces ICAD-S and ICAD-L mRNAs by alternative splicing. We have recently found that ICAD-L but not ICAD-S has an ability to work as a chaperone to enhance the correct folding of the CAD protein during CAD synthesis, although both ICAD-L and ICAD-S proteins efficiently inhibit the CAD' DNase activity. ${ }^{18}$ Whether ICAD-S has a specific function or not, remains to be determined.

Murine $C A D$ and ICAD mRNAs were expressed rather ubiquitously in various tissues, although its expression level in the lymphocytic tissues such as the thymus, spleen and lymph nodes was relatively high. Yet, the characteristics of the $5^{\prime}$ flanking promoter region were significantly different between the $C A D$ and ICAD genes. The promoter region of the murine $C A D$ gene was very $\mathrm{G}$ and $C$ rich as found in many ubiquitous house-keeping genes. On the other hand, the $\mathrm{G}$ and $\mathrm{C}$ content in the promoter region of murine ICAD was much lower than for $C A D$. The promoter analysis of the $C A D$ and ICAD genes indicated that the sequence up to -189 of the $C A D$ gene is sufficient to stimulate the transcription in HeLa and Jurkat cells. Several cis-regulatory elements for binding of various transcription factors such as RFX-1, SP-1, AP-2, $\mathrm{MZF}-1$ and Ets are in this region. The ICAD gene expression required the sequence between -118 and -8 of the ICAD gene, in which binding sites for Ikaros, cRel, Myc and Gfi-1 can be found. Except for MZF-1 and Ikaros, these transcription factors are rather ubiquitously expressed in various tissues, as found for $C A D$ and ICAD genes. Although it will be necessary to determine which transcription factors are responsible for the expression of the respective genes, these results suggest that expression of $C A D$ and ICAD genes are regulated by different mechanisms.

\section{Materials and Methods}

\section{Isolation of murine chromosomal genes for CAD and ICAD}

$4 \times 10^{5}$ plaques of $\lambda$ FIXII carrying a genomic DNA fragment from 129/ SV mice (Stratagene, La Jolla, CA, USA) were screened under high stringency conditions using a DNA fragment carrying murine $C A D$ or $I C A D-L C D N A^{8}$ as probes. Recombinant $\lambda D N A s$ that were positive with 
the probe were subcloned into pBluescript II, characterized by restriction enzyme mapping and $\mathrm{PCR}$ (polymerase chain reaction) using primers in exons. The DNA appropriate DNA fragments were then characterized by DNA sequencing analysis.

\section{Hybridization and DNA sequencing}

Colony, plaque, and Northern hybridizations were carried out as described previously. ${ }^{23}$ The probe DNA was prepared by PCR using the appropriate plasmid DNA as a template, and labeled with $\alpha^{3}{ }^{32} \mathrm{P}$ dCTP (New England Nuclear, Wilmington, DE, USA) using a random primer labeling kit (Boehringer Mannheim, Germany). DNA sequencing was carried out using a DNA sequencer (model 373A, Applied Biosystems, Foster City, CA, USA) and a Taq DyeDeoxy Terminator cycle sequencing kit (Applied Biosystems).

\section{5' RACE}

The 5'-RACE was carried out using a kit from Clontech Laboratories (Palo Alto, CA, USA) according to the protocol provided. In brief, the first-strand CDNA was synthesized using a primer (5'-CTGGCTCACATGATGTAGGAGATC-3') at the nucleotide position of 373-396 for $C A D$, and a primer ( $5^{\prime}$-GATAAACTCAGCTCTGG-3') at the nucleotide position of 751-767 for ICAD. The CAD- and ICAD-specific cDNAs were then amplified using a primer (ASO7; 5'-GGCCAGCAGCTTTTGCCTCAG-3') at the nucleotide position of $352-372$, and a primer (AS3; 5'-TCCACTTCTCATTGCAGGCC-3') at the nucleotide position of 264-283, respectively. The PCR products were cloned in pGEM ${ }^{\mathrm{R}}$-T Easy Vector (Promega, Madison, WI, USA), and their DNA sequences were determined.

\section{Interspecific murine backcross mapping}

Interspecific backcross progeny were generated by mating (C57BL/ $6 \mathrm{~J} \times M$. spretus) $\mathrm{F}_{1}$ females and $\mathrm{C} 57 \mathrm{BL} / 6 \mathrm{~J}$ males as described. ${ }^{15} \mathrm{~A}$ total of $205 \mathrm{~N}_{2}$ mice were used to map the Icad and Cad loci (see text for details). DNA isolation, restriction enzyme digestion, agarose gel electrophoresis, Southern blot transfer and hybridization were performed essentially as described. ${ }^{24}$ All blots were prepared with Hybond- $\mathrm{N}^{+}$nylon membrane (Amersham, Arlington Heights, IL, USA). The probe for Icad was an about $1.1 \mathrm{~kb}$ fragment of murine cDNA and the probe for $\mathrm{Cad}$ was an about $1.6 \mathrm{~kb}$ fragment of murine cDNA. The probes were labeled with $\alpha^{32} \mathrm{P}$-dCTP using a nick translation labeling kit (Boehringer Mannheim); washing was done to a final stringency of $0.8-1.0 \times$ SSCP, $0.1 \%$ SDS, $65^{\circ} \mathrm{C}$. Fragments of 21.0 and $3.6 \mathrm{~kb}$ were detected in Sphl digested C57BL/6J (B) DNA and a fragments of 5.3, 3.8 and $3.6 \mathrm{~kb}$ were detected in Sphl digested M. spretus (S) DNA using the Icad probe. Bg/ll fragments of $11.0,1.8$, and $1.5(B)$ and 15.0 and $2.6 \mathrm{~kb}(\mathrm{~S})$ were detected with $\mathrm{Cad}$ probe. The presence or absence of the M.spretus-specific fragments, which co-segregated for each probe, was followed in backcross mice.

A description of the probes and RFLPs for the loci linked to Icad and Cad including Htrld, Nppa and Gnb1 has been reported previously. ${ }^{25,26}$ Recombination distances were calculated using Map Manager, version 2.6.5. Gene order was determined by minimizing the number of recombination events required to explain the allele distribution patterns.

\section{Transfection and luciferase assay}

A series of fragments containing various length of the $5^{\prime}$ flanking region of murine $C A D$ and $I C A D$ genes were prepared by PCR. After confirming its authenticity by DNA sequencing, they were joined to the luciferase gene in PBPA-TATALuc vector, which contains a TATA box derived from murine G-CSF gene upstream of the firefly luciferase gene. ${ }^{27}$ Human HeLa and Jurkat cell lines were cultured in Dulbecco's modified Eagle's medium containing $10 \%$ fetal calf serum (FCS, Gibco BRL) or RPMI 1640 medium containing $10 \%$ FCS, respectively. HeLa cells $\left(5 \times 10^{3}\right.$ cells) were transfected with $500 \mathrm{ng}$ of the reporter plasmid by lipofection using spherical cationic polyamidoamine polymers (SuperFect Transfection Reagent, QIAGEN). As an internal control of the transfection, $100 \mathrm{ng}$ of pBOS-Rluc plasmid carrying the sea pansy luciferase gene (Promega) in pEF-BOS vector was included in each transfection. After culturing cells at $37^{\circ} \mathrm{C}$ for $24 \mathrm{~h}$, the luciferase activity in the cell lysates was measured using Dual Promoter Luciferase Assay Kit from Promega. The firefly luciferase activity of each sample was normalized to the sea pansy luciferase activity. Jurkat cells $\left(4 \times 10^{5}\right.$ cells) were transfected with $1 \mu \mathrm{g}$ of the reporter plasmid and $200 \mathrm{ng}$ of the pBOS-Rluc plasmid by the lipofection method using DIMREA-C Reagent from Gibco BRL. Cells were cultured at $37^{\circ} \mathrm{C}$ for $30 \mathrm{~h}$, then the luciferase activity in the cell lysates was measured as described above.

\section{Acknowledgements}

We thank Andreé Reuss and Deborah B. Householder for excellent technical assistance, and Ms. S. Kumagai for secretarial assistance. This work was supported, in part, by Grants-in-Aid from the Ministry of Education, Science, Sports, and Culture in Japan, and by the National Cancer Institute, DHHS, under contract with ABL.

\section{References}

1. Jacobson MD, Weil M and Raff MC (1997) Programmed cell death in animal development. Cell 88: 347-354

2. Wyllie AH, Kerr JFR and Currie AR (1980) Cell death: the significance of apoptosis. Int. Rev. Cytol. 68: 251-306

3. Wyllie AH (1980) Glucocorticoid-induced thymocyte apoptosis is associated with endogenous endonuclease activation. Nature 284: 555-556

4. Cohen GM (1997) Caspases: the executioners of apoptosis. Biochem. J. 326:116

5. Nicholson DW and Thornberry NA (1997) Caspases: killer proteases. Trends Biochem. Sci. 22: 299-306

6. Salvesen GS and Dixit VM (1997) Caspases: Intracellular signaling by proteolysis. Cell 91: 443-446

7. Thornberry NA and Lazebnik Y (1998) Caspases: enemies within. Science 281: $1312-1316$

8. Enari M, Sakahira H, Yokoyama H, Okawa K, Iwamatsu A and Nagata S (1998) A caspase-activated DNase that degrades DNA during apoptosis and its inhibitor ICAD. Nature 391: 43-50

9. Halenbeck R, MacDonald H, Roulston A, Chen TT, Conroy L and Williams LT (1998) CPAN, a human nuclease regulated by the caspase-sensitive inhibitor DFF45. Current Biology 8: 537-540

10 Liu X, Li P, Widlak P, Zou H, Luo X, Garrard WT and Wang X (1998) The 40-kDa subunit of DNA fragmentation factor induces DNA fragmentation and chromatin condensation during apoptosis. Proc. Natl. Acad. Sci. USA 95: 8461-8466

11 Mukae N, Enari M, Sakahira H, Fukuda Y, Inazawa J, Toh H and Nagata S (1998) Molecular cloning and characterization of human caspase-activated DNase. Proc. Natl. Acad. Sci. USA 95: 9123-9128

12. Sakahira $H$, Enari $M$ and Nagata $S$ (1998) Cleavage of CAD inhibitor in CAD activation and DNA degradation during apoptosis. Nature 391: $96-99$

13. Liu X, Zou H, Slaughter C and Wang X (1997) DFF, a heterodimeric protein that functions downstream of caspase-3 to trigger DNA fragmentation during apoptosis. Cell 89: 175-184

14. Inohara N, Koseki T, Chen S, Wu X and Nunez G (1998) CIDE, a novel family of cell death activators with homology to the $45 \mathrm{kDa}$ subunit of the DNA fragmentation factor. EMBO J. 17: 2526-2533 
15. Copeland NG and Jenkins NA (1991) Development and applications of a molecular genetic linkage map of the mouse genome. Trends Genet. 7:113-118

16. Breathnach Rand Chambon $P$ (1981) Organization and expression of eukaryotic split genes coding for proteins. Ann. Rev. Biochem. 50: 349-383

17. Leek JP, Carr IM, Bell SM, Markham AF and Lench NJ (1997) Assignment of the DNA fragmentation factor gene (DFFA) to human chromosome bands 1p36.3p36.2 by in situ hybridization. Cytogenet. Cell Genetics 79: 212-213

18. Sakahira H, Enari M and Nagata S (1999) Functional differences of two forms of the inhibitor of caspase-activated DNase, ICAD-L and ICAD-S. J. Biol. Chem. 274: $15740-15744$

19. Aravind L, Dixit VM and Koonin EV (1999) The domains of death: evolution of the apoptosis machinery. Trends Biochem. Sci. 24: 47-53

20. Hofmann K, Bucher P and Tschopp J (1997) The CARD domain: a new apoptotic signaling motif. Trends Biochem. Sci. 22: 155-166

21. Wallach D (1997) Cell death induction by TNF: a matter of self control. Trends Biochem. Sci. 22: 107-109

22. Mclloy D, Sakahira T, Talanian RV and Nagata S (1999) Involvement of caspase 3-activated DNase in internucleosomal DNA cleavage induced by diverse apoptotic stimuli. Oncogene in press

23. Sambrook J, Fretsch EF and Maniatis T (1989) Molecular cloning: A laboratory manual, Cold Spring Harbor, NY, Cold Spring Harbor Laboratory
24. Jenkins NA, Copeland NG, Taylor BA and Lee BK (1982) Organization, distribution, and stability of endogenous ecotropic murine leukemia virus DNA sequences in chromosomes of Mus musculus. J. Virol. 43: 26-36

25. Birekeland ML, Copeland NG, Gilbert DJ, Jenkins NA and Barclay AN (1995) Gene structure and chromosomal localization of the mouse homologue of rat OX40 protein. Eur. J. Immunol. 25: 926-930

26. Wilkie TM, Chen Y, Gilbert DJ, Moore KJ, Yu L, Simon MI, Copeland NG and Jenkins NA (1993) Identification, chromosomal location, and genome organization of mammalian G-protein-coupled receptors. Genomics 18: 175184

27. Murai K, Murakami H and Nagata S (1998) Myeloid-specific transcriptional activation by murine myeloid zinc finger protein-2. Proc. Natl. Acad. Sci. USA 95: 3461-3466

28. Suda T, Takahashi T, Golstein P and Nagata S (1993) Molecular cloning and expression of the Fas ligand, a novel member of the tumor necrosis factor family. Cell 75: $1169-1178$

29. Quandt K, Frech K, Karas H, Wingender E and Werner T (1995) MatInd and Matlnspector - New fast and versatile tools for detection of consensus matches in nucleotide sequence data. Nucleic Acids Res. 23: 4878-4884 